\title{
nDsbD: A redox interaction hub in the Escherichia coli periplasm
}

\section{Review Article}

Author(s):

Stirnimann, Christian U.; Grütter, Markus G.; Glockshuber, Rudi; Capitani, Guido

Publication date:

2006-07

Permanent link:

https://doi.org/10.3929/ethz-b-000036278

Rights / license:

In Copyright - Non-Commercial Use Permitted

Originally published in:

Cellular and Molecular Life Sciences 63(14), https://doi.org/10.1007/s00018-006-6055-1 


\title{
Review
}

\section{nDsbD: a redox interaction hub in the Escherichia coli periplasm}

\author{
C. U. Stirnimann ${ }^{\text {a }}$, M. G. Grütter ${ }^{\text {a, *, R. Glockshuber }}{ }^{\text {b }}$ and G. Capitani ${ }^{\text {a , * }}$, \\ ${ }^{a}$ Biochemisches Institut, Universität Zürich, Winterthurerstrasse 190, 8057 Zürich (Switzerland), \\ Fax: +41 44635 6834, e-mail: gruetter@bioc.unizh.ch, capitani@bioc.unizh.ch \\ ${ }^{\mathrm{b}}$ Institut für Molekularbiologie und Biophysik, Eidgenössische Technische Hochschule Hönggerberg, 8093 Zürich \\ (Switzerland)
}

Received 3 February 2006; received after revision 1 March 2006; accepted 5 April 2006

Online First 19 June 2006

\begin{abstract}
DsbD is a redox-active protein of the inner Escherichia coli membrane possessing an N-terminal $(\mathrm{nDsbD})$ and a C-terminal (cDsbD) periplasmic domain. $\mathrm{nDsbD}$ interacts with four different redox proteins involved in the periplasmic disulfide isomerization and in the cytochrome $c$ maturation systems. We review here the studies that led to the structural characterization of all
\end{abstract}

soluble DsbD domains involved and, most importantly, of trapped disulfide intermediate complexes of $\mathrm{nDsbD}$ with three of its four redox partners. These results revealed the structural features enabling nDsbD, a 'redox hub' with an immunoglobulin-like fold, to interact efficiently with its different thioredoxin-like partners.

Keywords. Disulfide isomerization, cytochrome $c$ maturation, DsbD, DsbC, DsbG, CcmG.

\section{The disulfide bond formation and isomerization pathways of $E$. coli}

Disulfide bond formation is a very important post-translational modification of secretory proteins. In Gram-negative bacteria, such as Escherichia coli, disulfide bond formation and isomerization are catalyzed in the periplasm by members of the Dsb protein family [1-3]. The two types of reaction, disulfide bond formation and isomerization, are coupled to independent pathways (Fig. 1). The disulfide formation pathway consists of two proteins, DsbA and DsbB. Oxidized DsbA, possessing a thioredoxin-like fold with a helical insertion [4], introduces disulfide bonds to non-native substrate proteins in a random and very fast manner $[5,6]$. The inner membrane protein DsbB re-oxidizes DsbA and keeps it in its active

* Corresponding authors. state [7]. The two electrons that flow from reduced DsbA to $\mathrm{DsbB}$ are then passed from DsbB to ubiquinone- 8 from the respiratory chain $[7,8]$.

DsbA only possesses a very low disulfide isomerase activity. Non-native disulfide bonds introduced by DsbA into substrate proteins are rearranged by DsbC, an efficient periplasmic disulfide isomerase with chaperone activity [9]. DsbC, which does not react with DsbA [10, $11]$, is kept in its catalytically active, reduced state by the inner membrane protein DsbD [12], which consists of a central transmembrane domain (tDsbD) with eight predicted transmembrane helices, an immunoglobulin-like N-terminal (nDsbD) and a thioredoxin-like C-terminal periplasmic domain (cDsbD) [13-18]. Each of these three domains contains one pair of invariant cysteines, which are crucial for disulfide exchange [13]. DsbD functions as a reductant, receiving its reducing power from cytoplasmic NADPH (reduced nicotinamide adenine dinucleotide phosphate). Two electrons are transferred from NADPH 
via thioredoxin reductase to thioredoxin $[19,20]$, which passes them on to tDsbD. Electrons are then further transferred via cDsbD to $\mathrm{nDsbD}$ [21-23], which can be viewed as a 'redox hub' that distributes electrons to several periplasmic target proteins. Besides DsbC, in vivo substrates of $\mathrm{nDsbD}$ are $\mathrm{DsbG}$ and $\mathrm{CcmG}$, which, like DsbC, both belong to the thioredoxin superfamily [21, 24]. DsbG is a putative DsbC paralogue with so far unknown cellular function, exhibiting low disulfide isomerase activity and chaperone activity [25-27].

The third substrate, $\mathrm{CcmG}$, is a periplasmic, membraneanchored protein belonging to the cytochrome $c$ maturation pathway. $\mathrm{CcmG}$ is thought to activate $\mathrm{CcmH}$, which itself reduces apocytochrome $c$, enabling it to become active and incorporate heme [28-30].

In the last few years, the $\mathrm{DsbD} / \mathrm{DsbC}$ and $\mathrm{DsbD} / \mathrm{CcmG}$ redox systems have been characterized in detail through structural and functional studies. The present review summarizes the current knowledge regarding the structures and interactions of the various components. The analysis highlights the central role of $\mathrm{nDsbD}$ as interaction hub, and in particular the question of how $\mathrm{nDsbD}$ specifically interacts with four different components of the disulfide isomerization and cytochrome $c$ maturation pathways.

\section{The disulfide isomerase DsbC and its homologue, DsbG}

DsbC was the first structurally characterized component of the bacterial protein disulfide isomerization pathway [31]. DsbC is a V-shaped homodimer, with each monomer containing an N-terminal dimerization domain (aa1-aa61) and a C-terminal catalytic domain (aa78-aa216), connected by a linker $\alpha$-helix. The catalytic domain can be divided into two subdomains. The first one (encompassing aa78-aa122 and aa167-aa216) possesses a thioredoxin-like fold and contains two cysteines (Cys98 and Cys101) that are necessary for activity. The second subdomain (aa123-aa166; $\alpha$-helices 4 and 5) carries an insertion into the thioredoxinlike fold at the same position as found in DsbA [31]. The overall arrangement of the dimer (Fig. 2a) is such that a large hydrophobic cleft separates the two active sites. McCarthy et al. [31] proposed that this cleft represents the binding site for protein substrates and is required for the chaperone and isomerase activity of DsbC. Dimerization of DsbC is crucial for its isomerase activity [32] and prevents it from interacting with DsbB [33]. In addition, dimerization of DsbC increases its affinity towards its reductant, nDsbD [17, 34], in perfect agreement with the structural finding that each DsbC subunit contributes one interface to the complex with $\mathrm{nDsbD}$.

Recently, also the structure of DsbG, a protein sharing $24 \%$ sequence identity with DsbC, was solved [24, 31, 35]. Like DsbC, DsbG exhibits a V-shaped homodimeric structure, but of larger size. This is essentially due to the fact that DsbG has a longer linker helix (2.5 additional turns) between the thioredoxin-like and dimerization domains. In addition, the DsbG dimer possesses a cleft of nearly double the size compared with that of DsbC. The cleft surface of DsbG is also partially hydrophobic,

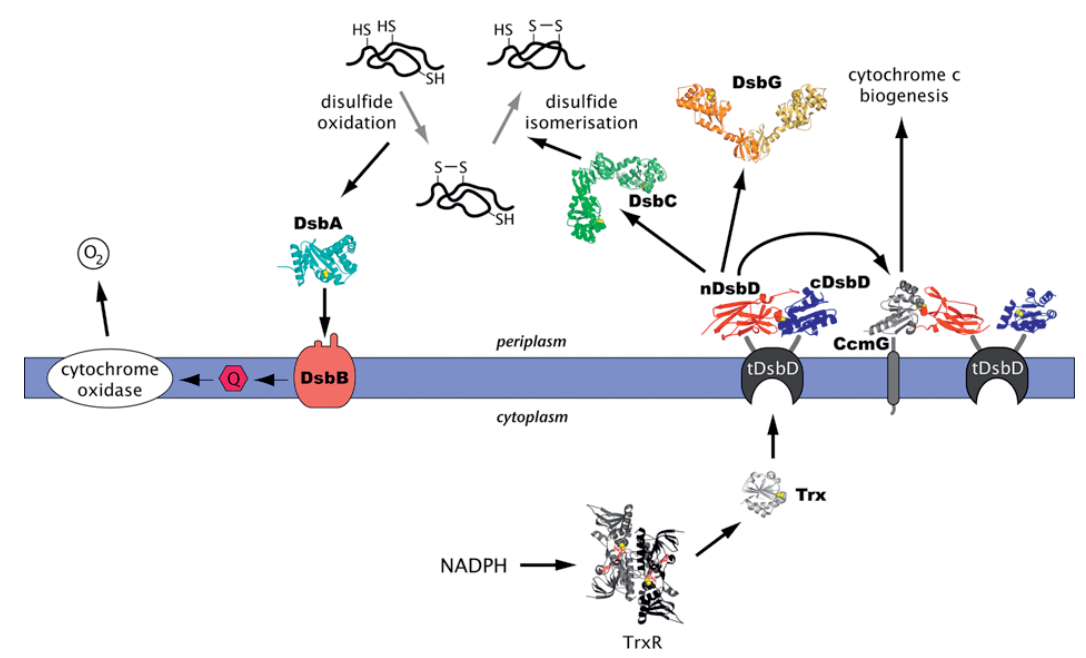

Figure 1. An overview of the E. coli Dsb-system. Two electrons are transported from cytoplasmic NADPH via thioredoxin reductase (dark grey and black; PDB-code: 1CL0 [44]) and thioredoxin (light grey; 2TRX [45]) to tDsbD. tDsbD transports the electrons through the membrane via periplasmic cDsbD (blue; 1VRS [10] and 2FWE [39]) to $\mathrm{nDsbD}$ (red; 1VRS [10] and 1Z5Y [40]). nDsbD functions as a hub protein and passes the electrons on to either DsbC (green; 1EEJ [31]; disulfide isomerization pathway) or CcmG (grey; 1Z5Y [40]; cytochrome $c$ maturation) or DsbG (orange; 1v58 [35]). DsbC isomerizes non-native disulfide bonds to native disulfide bonds. DsbA (cyan; 1DSB [4]) randomly oxidizes unfolded polypeptide chains and transports the electrons to DsbB (pink) that itself gives them via quinone (claret-red) and cytochrome oxidases to molecular oxygen. A flow of two electrons is indicated by black arrows. 
a
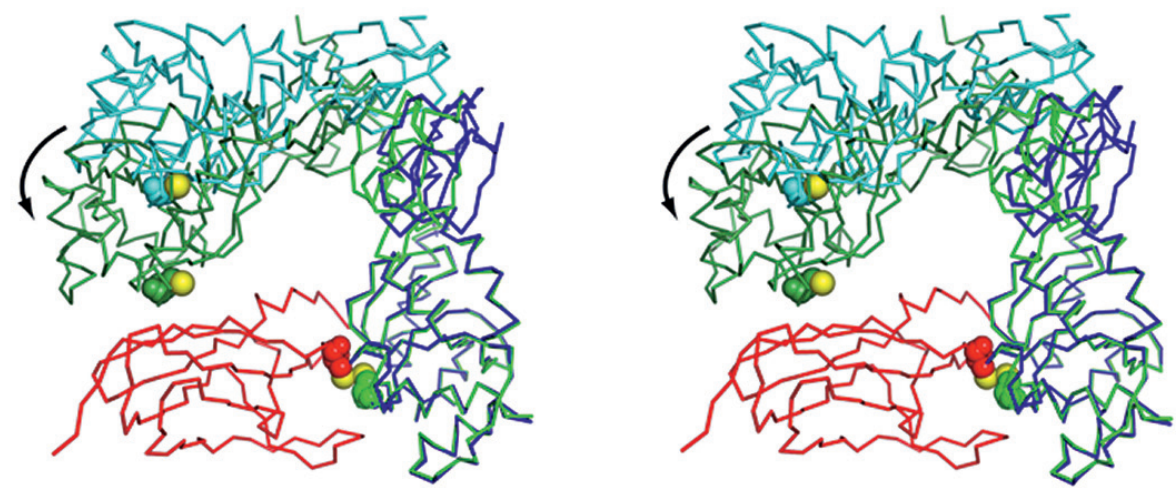

b

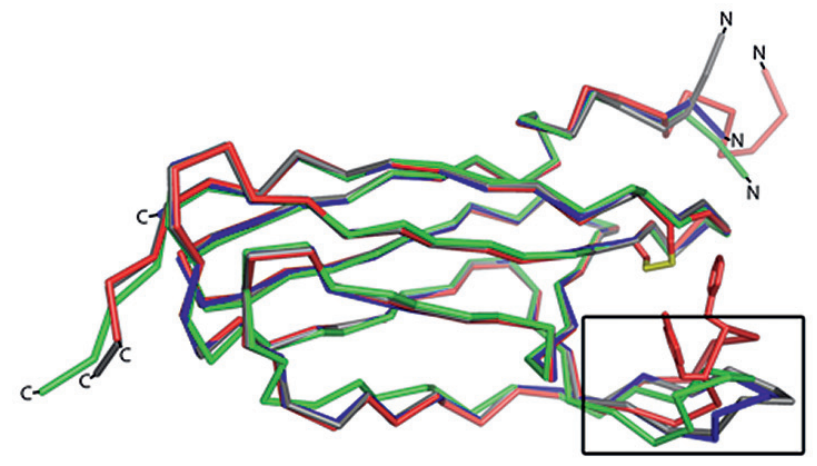

C
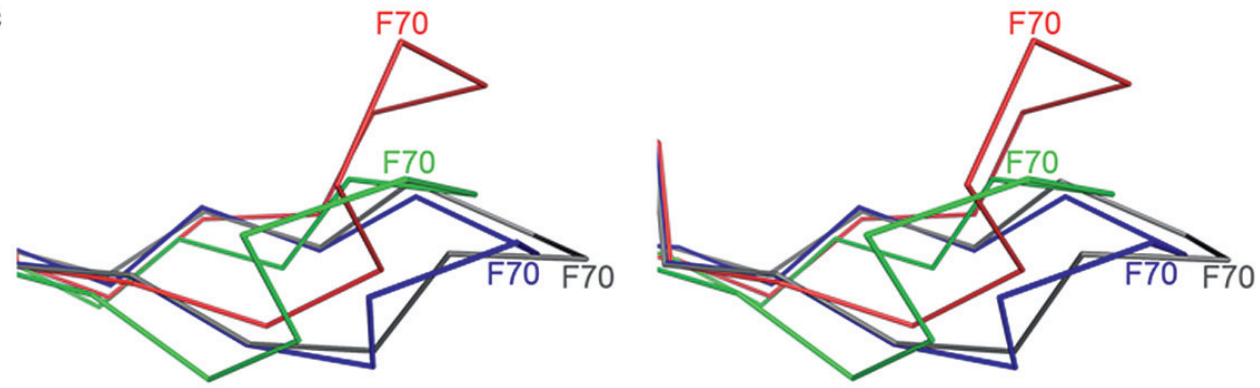

Figure 2. (a) Uncomplexed dimeric DsbC (blue and cyan; 1EEJ [31]) undergoes a large conformational change upon complex formation with $\mathrm{nDsbD}$ (light and dark green (DsbC) and red (nDsbD); 1JZD [17]). The solvent-accessible cysteines are depicted in spacefill representation with the $\mathrm{S}_{\gamma}$ atom coloured in yellow. (b) Superposition of the different $\mathrm{nDsbD}$ structures available. The $\mathrm{C}_{\alpha}$ trace of uncomplexed nDsbD is depicted in red, the Cys103-Cys109 disulfide bond and the Phe70 and Tyr71 side chains (which shield the disulfide bridge) appear in stick representation (1L6P [16]). The $\mathrm{C}_{\alpha}$ trace of $\mathrm{nDsbD}$ in $\mathrm{nDsbD}-\mathrm{SS}-\mathrm{DsbC}$ is coloured in green (1JZD [17]), the one of $\mathrm{nDsbD}$ in $\mathrm{nDsbD}-\mathrm{SS}-\mathrm{cDsbD}$ in blue (1VRS [10]) and the one of $\mathrm{nDsbD}$ in nDsbD-SS-CcmG in grey (1Z5Y [40]). (c) Zoomed stereoview (frame in Fig. $2 b$ ), showing the differences in the opening of the $\mathrm{nDsbD}$ cap-loop region. In $\mathrm{nDsbD-SS}-\mathrm{cDsbD}$ (blue) and $\mathrm{nDsbD-SS-CcmG} \mathrm{(grey)}$ the cap-loop undergoes a clear opening compared with uncomplexed nDsbD (red), whereas in nDsbD-SS-DsbC the less opened cap-loop (green) is laterally shifted.

but also features negatively charged patches that are not found in DsbC [35].

\section{nDsbD: activator of the disulfide bond isomerase DsbC}

Initial bioinformatics studies on DsbD suggested that its $\mathrm{cDsbD}$ domain might belong to the thioredoxin-like su- perfamily, due to the presence of a typical CXXC active site motif and due to the sequence similarity of $\mathrm{cDsbD}$ to the thioredoxin-like domains of protein disulfide isomerase (PDI) [36-38]. The central region of the DsbD sequence could be readily assigned by bioinformatics and experimental topological analyses to a transmembrane domain with eight predicted transmembrane helices [1315]. In contrast, the topology of $\mathrm{nDsbD}$ remained unclear in the absence of a crystal structure. The sequence of 
nDsbD does not contain the typical CXXC thioredoxin motif, but a $\mathrm{CX}_{5} \mathrm{C}$ motif instead. In 2002, two groups independently solved the structure of nDsbD: Goulding et al. [16] determined the crystal structure of the unliganded protein, while shortly after Haebel et al. [17] published a comprehensive structural study on the kinetically stabilized, mixed disulfide complex between $\mathrm{nDsbD}$ and the DsbC homodimer (nDsbD-SS-DsbC).

Unlike the other Dsb proteins, namely DsbA, DsbC, $\mathrm{CcmG}$ and $\mathrm{cDsbD}$, which all possess a thioredoxinlike fold, $\mathrm{nDsbD}$ features an immunoglobulin-like fold consisting of a $\beta$-sandwich formed by two antiparallel $\beta$-sheets. The active site cysteines Cys103 and Cys109 connect two neighbouring $\beta$-strands in the oxidized state of $\mathrm{nDsbD}$. In unliganded nDsbD, the so-called cap-loop region (residues Asp68-Gly72) protects the active site cysteines from the environment and assumes a closed conformation, disfavouring illegitimate redox reactions (Fig. 2b, c) [16, 17].

Upon formation of a mixed disulfide between $\mathrm{nDsbD}$ and DsbC, the cap-loop of nDsbD opens (Fig. 2b, c), and the V-shaped DsbC dimer adopts a more closed conformation to form the nDsbD-SS-DsbC complex (Fig. 2a). As already mentioned, the complex possesses two interfaces. In the first one (primary interface), Cys109 of nDsbD, forms an intermolecular disulfide bond with Cys98 of one $\mathrm{DsbC}$ monomer. The second subunit of DsbC forms a secondary, asymmetric binding interface with the opposite side of $\mathrm{nDsbD}$. The active site of the second $\mathrm{DsbC}$ subunit is involved in the secondary binding interface, but it is not covalently linked to $\mathrm{nDsbD}$, which does not possess any cysteines in addition to those of the active site [17].

\section{Different modes of interaction in nDsbD-SS-cDsbD and nDsbD-SS-DsbC}

The C-terminal domain of DsbD has also been structurally characterized. With a $\mathrm{C}_{\alpha}$-rmsd of $1.54 \AA$ for 91 out of 105 residues, its structure shows a high similarity to $E$. coli thioredoxin and it functions as a stiff electron shuttle between $\mathrm{tDsbD}$ and $\mathrm{nDsbD}[18,39]$. Upon complex formation of $\mathrm{nDsbD}$ and $\mathrm{cDsbD}$ (nDsbD-SS-cDsbD), cDsbD exhibits only one significant conformational change: the main chain carbonyl of Thr529, forced by van der Waals interactions exerted by Phe11 and Phe108 of $\mathrm{nDsbD}$ [39], rotates by about $100^{\circ}$. The cap-loop region of $\mathrm{nDsbD}$ adopts an even more open conformation than that observed for nDsbD-SS-DsbC (Fig. 2b, c). By superimposing the $\mathrm{nDsbD}$ molecules of the two complexes, the relative orientations of the thioredoxin-like folds of cDsbD and DsbC, respectively, differ by around $20^{\circ}$. In the $\mathrm{nDsbD}-\mathrm{SS}-\mathrm{cDsbD}$ complex, cDsbD shares only one binding interface with $\mathrm{nDsbD}$ compared with the two

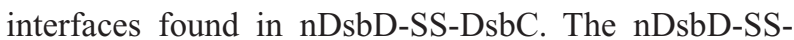
$\mathrm{cDsbD}$ interface and the primary interface of $\mathrm{nDsbD}$ SS-DsbC have a similar size of around $1300 \AA^{2}$, but the total binding interface area of nDsbD-SS-DsbC is about 1.5 -fold larger due to the second interface. These findings imply a lower affinity of $\mathrm{nDsbD}$ to $\mathrm{cDsbD}$ than to DsbC, which can, however, be compensated by high effective concentrations of $\mathrm{nDsbD}$ and $\mathrm{cDsbD}$ in the context of the full-length protein.

The bimolecular reduction of $\mathrm{nDsbD}$ by $\mathrm{cDsbD}$ and DsbC by nDsbD are both very fast, with rate constants of $1.1 \cdot 10^{5} \mathrm{M}^{-1} \mathrm{~s}^{-1}$ and $3.9 \cdot 10^{6} \mathrm{M}^{-1} \mathrm{~s}^{-1}[10]$.

Modelling of the nDsbD-SS-DsbG complex on the basis of the known structure of DsbG and the primary interface between $\mathrm{nDsbD}$ and $\mathrm{DsbC}$ showed that the cleft in DsbG, in principle, was wide enough to allow two copies of $\mathrm{nDsbD}$ to form a mixed disulfide complex with each subunit of DsbG without steric clashes. Whether both subunits of DsbG can be reduced simultaneously by two molecules of DsbD in vivo remains unknown, but the analysis indicates that a secondary interface between $\mathrm{nDsbD}$ and the second subunit of DsbG is unlikely.

\section{nDsbD reduces CcmG: branching to another redox pathway}

$\mathrm{CcmG}$ is the third interaction partner for which a structure in a mixed-disulfide complex with $\mathrm{nDsbD}$ is known [40]. High-resolution crystal structures of uncomplexed CcmG had been described earlier for the Gram-negative bacterium Bradirhyzobium japonicum and the related protein of the Gram-positive Mycobacterium tuberculosis [41, 42]. B. japonicum $\mathrm{CcmG}$ possesses a thioredoxin-like fold, enriched by an $\alpha \beta$-insertion (after $\beta$-strand 2) that is required for $c$-type cytochrome maturation. Additionally, an N-terminal extension links the transmembrane anchor helix to the thioredoxin-like domain [41]. With a low rmsd value of $1.4 \AA$ (on $\mathrm{C}_{\alpha}$-atoms), B. japonicum $\mathrm{CcmG}$ is very similar to $E$. coli $\mathrm{CcmG}$ (from the $\mathrm{nDsbD}$ SS-CcmG complex), and only the seven-residue-shorter $\mathrm{N}$-terminal extension adopts a different conformation [40].

$\mathrm{nDsbD}$ interacts with $\mathrm{CcmG}$ in a manner similar to what it does with cDsbD: (i) the relative orientation of the thioredoxin-like domains is the same and (ii) the cap-loop region opens in a similar way upon complex formation (Fig. 2b, c), but exhibits more flexibility as judged from the features of the electron density in the crystal structure and from the temperature factors of the refined atomic model. An important feature found neither in the nDsbDSS-cDsbD nor in the nDsbD-SS-DsbC complex is a substantial involvement of $\mathrm{N}$-terminal $\mathrm{nDsbD}$ residues (Ser9 and Phe11) in the binding interface. These residues form 
a hydrogen-bond network with Tyr141 of CcmG (a highly conserved residue among different species), the phenolic ring of which fits in a hydrophobic pocket formed by residues of $\mathrm{CcmG}$ and $\mathrm{nDsbD}$. In contrast, $\mathrm{cDsbD}$ and DsbC do not have a tyrosine at that position and do not specifically interact with these $\mathrm{N}$-terminal $\mathrm{nDsbD}$ residues [40].

\section{The hub function of nDsbD}

$\mathrm{nDsbD}$ is a very interesting subject for analysis of proteinprotein interactions, since it has been structurally characterized both in the unliganded state and in complex with three of the four known in vivo partners (cDsbD, DsbC and $\mathrm{CcmG}$ ). In the three complexes (and considering only the primary interface for $\mathrm{nDsbD}-\mathrm{SS}-\mathrm{DsbC}$ ), around $90 \%$ of the $\mathrm{nDsbD}$ interface residues (contacting the active site of the partner protein) are the same. In all three cases the interface is rather small (area around $1350 \AA^{2}$ ), and mediates only a small number of interactions. The adaptability of $\mathrm{nDsbD}$ to three different targets results from at least three structural features: (i) it possesses a very flexible cap-loop region, which assumes a closed conformation in the absence of a protein ligand. Upon formation of the mixed disulfide, the cap-loop region adopts a more open conformation, adapting to the partner protein. (ii) For $\mathrm{DsbC}$ recognition, $\mathrm{nDsbD}$ employs a second, flat binding interface on its side. In this case, interactions are of noncovalent nature and include one salt bridge. (iii) The Nterminal segment of $\mathrm{nDsbD}$ plays a role in the recognition of CcmG: it is involved in a hydrogen-bonded network and in hydrophobic interactions with Tyr 141 of CcmG, a residue not conserved in the two other partners of $\mathrm{nDsbD}$ (it corresponds to a Ser180 in DsbC and to a Leu508 in cDsbD).

In contrast to $\mathrm{nDsbD}$, two of the three thioredoxin-like target proteins of $\mathrm{nDsbD}[\mathrm{cDsbD}$ and $\mathrm{CcmG}$, based on a comparison between CcmGs of $E$. coli (complex) and $B$. japonicum] undergo very small conformational changes upon formation of the mixed disulfide complex with $\mathrm{nDsbD}[10,40]$. In complexed DsbC, the only conformational change involves the relative orientation of

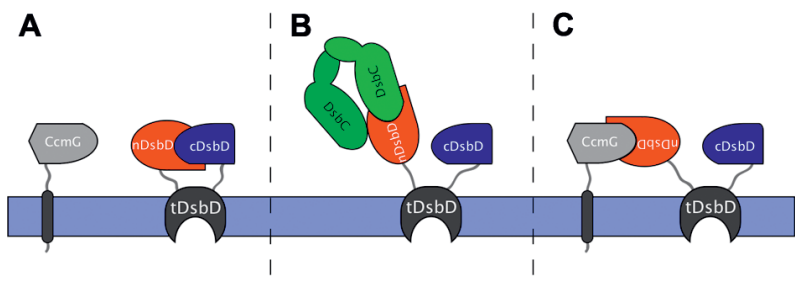

Figure 3. Schematic illustration of proposed reorientations of nDsbD to fulfil its 'redox hub' function $[10,40]$. Panel $A$ shows the proposed orientation in the nDsbD-SS-cDsbD complex; panels $B$ and $C$ depict possible orientations in the nDsbD-SS-DsbC and $\mathrm{nDsbD}-\mathrm{SS}-\mathrm{CcmG}$ complexes, respectively. the two subunits and leaves the thioredoxin domain unaffected [17].

Structural analysis of the three complexes prompted Rozhkova et al. [10] and Stirnimann et al. [40] to propose that $\mathrm{nDsbD}$, in order to form mixed disulfides with its various target proteins, must be able to undergo substantial movements relative to tDsbD. A likely orientation for two membrane-linked complexes, nDsbD-SS-cDsbD and $\mathrm{nDsbD}-\mathrm{SS}-\mathrm{CcmG}$, relative to membrane was proposed $[10,40]$. Based on that assumption, $\mathrm{nDsbD}$ has to substantially change its orientation relative to the membrane upon changing partner from $\mathrm{cDsbD}$ to $\mathrm{CcmG}$ (nDsbD turned 'up-side down'; see Fig. 3, panels A and C). Upon formation of the complex with the non membrane-bound $\mathrm{DsbC}, \mathrm{nDsbD}$ has to assume yet a different orientation to allow a proper interaction with both interfaces of DsbC (Fig. 3). The above observations underline the key function of $\mathrm{nDsbD}$ as a redox hub in the periplasm of Gramnegative bacteria.

\section{Concluding remarks}

All known periplasmic proteins interacting with $\mathrm{nDsbD}$ have been structurally characterized, as have the complexes of $\mathrm{nDsbD}$ with those partners, with the single exception of nDsbD-SS-DsbG. In spite of these successes, the transmembrane domain (tDsbD), to which $\mathrm{nDsbD}$ is anchored, remains elusive not only from a structural but also from a functional point of view: it is still unclear how electrons are transported through the membrane and how cytoplasmic thioredoxin or periplasmic cDsbD interact with $\mathrm{tDsbD}$ [43]. Thus, important research goals for the future are the structure determination of $\mathrm{tDsbD}$ and its complexes with soluble partners (cDsbD and thioredoxin), as well as the structure determination of the nDsbD-SS-DsbG complex.

Acknowledgements. This work was supported by the Schweizerische Nationalfonds, the ETH Zurich and the University of Zurich within the framework of the NCCR Structural Biology program. The authors would like to acknowledge their colleagues who contributed to the papers from the M. G. G. and R. G. groups quoted in this review.

1 Kadokura, H., Katzen, F. and Beckwith, J. (2003) protein disulfide bond formation in prokaryotes. Annu. Rev. Biochem. 72, 111-135.

2 Nakamoto, H. and Bardwell, J. C. (2004) Catalysis of disulfide bond formation and isomerization in the Escherichia coli periplasm. Biochim. Biophys. Acta 1694, 111-119.

3 Hiniker, A. and Bardwell, J. C. (2003) Disulfide bond isomerization in prokaryotes. Biochemistry 42, 1179-1185.

4 Martin, J. L., Bardwell, J. C. and Kuriyan, J. (1993) Crystal structure of the DsbA protein required for disulphide bond formation in vivo. Nature 365, 464-468.

5 Wunderlich, M., Otto, A., Seckler, R. and Glockshuber, R. (1993) Bacterial protein disulfide isomerase: efficient catalysis of oxidative protein folding at acidic $\mathrm{pH}$. Biochemistry 32 , $12251-12256$. 
6 Darby, N. J. and Creighton, T. E. (1995) Catalytic mechanism of Dsba and its comparison with that of protein disulfide-isomerase. Biochemistry 34, 3576-3587.

7 Bader, M., Muse, W., Ballou, D. P., Gassner, C. and Bardwell, J. C. (1999) Oxidative protein folding is driven by the electron transport system. Cell 98, 217-227.

8 Bader, M. W., Xie, T., Yu, C. A. and Bardwell, J. C. (2000) Disulfide bonds are generated by quinone reduction. J. Biol. Chem. 275, 26082-26088.

9 Chen, J., Song, J. L., Zhang, S., Wang, Y., Cui, D. F. and Wang, C. C. (1999) Chaperone activity of DsbC. J. Biol. Chem. 274, 19601-19605.

10 Rozhkova, A., Stirnimann, C. U., Frei, P., Grauschopf, U., Brunisholz, R., Grutter, M. G., Capitani, G. and Glockshuber, R. (2004) Structural basis and kinetics of inter- and intramolecular disulfide exchange in the redox catalyst DsbD. EMBO J. 23, 1709-1719.

11 Zapun, A., Missiakas, D., Raina, S. and Creighton, T. E. (1995) Structural and functional characterization of Dsbc, a protein involved in disulfide bond formation in Escherichia coli. Biochemistry $34,5075-5089$.

12 Porat, A., Cho, S. H. and Beckwith, J. (2004) The unusual transmembrane electron transporter DsbD and its homologues: a bacterial family of disulfide reductases. Res. Microbiol. 155, 617-622.

13 Stewart, E. J., Katzen, F. and Beckwith, J. (1999) Six conserved cysteines of the membrane protein DsbD are required for the transfer of electrons from the cytoplasm to the periplasm of Escherichia coli. EMBO J. 18, 5963-5971.

14 Chung, J., Chen, T. and Missiakas, D. (2000) Transfer of electrons across the cytoplasmic membrane by DsbD, a membrane protein involved in thiol-disulphide exchange and protein folding in the bacterial periplasm. Mol. Microbiol. 35, 1099 1109 .

15 Gordon, E. H., Page, M. D., Willis, A. C. and Ferguson, S. J. (2000) Escherichia coli DipZ: anatomy of a transmembrane protein disulphide reductase in which three pairs of cysteine residues, one in each of three domains, contribute differentially to function. Mol. Microbiol. 35, 1360-1374.

16 Goulding, C. W., Sawaya, M. R., Parseghian, A., Lim, V., Eisenberg, D. and Missiakas, D. (2002) Thiol-disulfide exchange in an immunoglobulin-like fold: structure of the N-terminal domain of DsbD. Biochemistry 41, 6920-6927.

17 Haebel, P. W., Goldstone, D., Katzen, F., Beckwith, J. and Metcalf, P. (2002) The disulfide bond isomerase DsbC is activated by an immunoglobulin-fold thiol oxidoreductase: crystal structure of the DsbC-DsbD $\alpha$ complex. EMBO J. 21, 4774-4784.

18 Kim, J. H., Kim, S. J., Jeong, D. G., Son, J. H. and Ryu, S. E. (2003) Crystal structure of DsbD $\gamma$ reveals the mechanism of redox potential shift and substrate specificity. FEBS Lett. 543, 164-169.

19 Rietsch, A., Belin, D., Martin, N. and Beckwith, J. (1996) An in vivo pathway for disulfide bond isomerization in Escherichia coli. Proc. Natl. Acad. Sci. USA 93, 13048-13053.

20 Rietsch, A., Bessette, P., Georgiou, G. and Beckwith, J. (1997) Reduction of the periplasmic disulfide bond isomerase, DsbC, occurs by passage of electrons from cytoplasmic thioredoxin. J. Bacteriol. 179, 6602-6608.

21 Katzen, F. and Beckwith, J. (2000) Transmembrane electron transfer by the membrane protein DsbD occurs via a disulfide bond cascade. Cell 103, 769-779.

22 Krupp, R., Chan, C. and Missiakas, D. (2001) DsbD-catalyzed transport of electrons across the membrane of Escherichia coli. J. Biol. Chem. 276, 3696-3701.

23 Collet, J. F., Riemer, J., Bader, M. W. and Bardwell, J. C. (2002) Reconstitution of a disulfide isomerization system. J. Biol. Chem. 277, 26886-26892.

24 Bessette, P. H., Cotto, J. J., Gilbert, H. F. and Georgiou, G. (1999) In vivo and in vitro function of the Escherichia coli periplasmic cysteine oxidoreductase DsbG. J. Biol. Chem. 274, 7784-7792.

25 Andersen, C. L., Matthey-Dupraz, A., Missiakas, D. and Raina, S. (1997) A new Escherichia coli gene, dsbG, encodes a periplasmic protein involved in disulphide bond formation, required for recycling DsbA/DsbB and DsbC redox proteins. Mol. Microbiol. 26, 121-132.

26 Shao, F., Bader, M. W., Jakob, U. and Bardwell, J. C. (2000) $\mathrm{DsbG}$, a protein disulfide isomerase with chaperone activity. $\mathrm{J}$. Biol. Chem. 275, 13349-13352.

27 Tan, J. T. and Bardwell, J. C. (2004) Key players involved in bacterial disulfide-bond formation. Chembiochem 5, 1479-1487.

28 Fabianek, R. A., Hennecke, H. and Thony-Meyer, L. (1998) The active-site cysteines of the periplasmic thioredoxin-like protein CcmG of Escherichia coli are important but not essential for cytochrome c maturation in vivo. J. Bacteriol. 180, 1947-1950.

29 Fabianek, R. A., Hofer, T. and Thony-Meyer, L. (1999) Characterization of the Escherichia coli $\mathrm{CcmH}$ protein reveals new insights into the redox pathway required for cytochrome c maturation. Arch. Microbiol. 171, 92-100.

30 Reid, E., Cole, J. and Eaves, D. J. (2001) The Escherichia coli $\mathrm{CcmG}$ protein fulfils a specific role in cytochrome $\mathrm{c}$ assembly. Biochem. J. 355, 51-58.

31 McCarthy, A. A., Haebel, P. W., Torronen, A., Rybin, V., Baker, E. N. and Metcalf, P. (2000) Crystal structure of the protein disulfide bond isomerase, DsbC, from Escherichia coli. Nat. Struct. Biol. 7, 196-199.

32 Sun, X. X. and Wang, C. C. (2000) The N-terminal sequence (residues 1-65) is essential for dimerization, activities, and peptide binding of Escherichia coli DsbC. J. Biol. Chem. 275, 22743-22749.

33 Bader, M. W., Hiniker, A., Regeimbal, J., Goldstone, D., Haebel, P. W., Riemer, J., Metcalf, P. and Bardwell, J. C. (2001) Turning a disulfide isomerase into an oxidase: DsbC mutants that imitate DsbA. EMBO J. 20, 1555-1562.

34 Goldstone, D., Haebel, P. W., Katzen, F., Bader, M. W., Bardwell, J. C., Beckwith, J. and Metcalf, P. (2001) DsbC activation by the N-terminal domain of DsbD. Proc. Natl. Acad. Sci. USA 98, 9551-9556.

35 Heras, B., Edeling, M. A., Schirra, H. J., Raina, S. and Martin, J. L. (2004) Crystal structures of the DsbG disulfide isomerase reveal an unstable disulfide. Proc. Natl. Acad. Sci. USA 101, 8876-8881.

36 Missiakas, D., Schwager, F. and Raina, S. (1995) Identification and characterization of a new disulfide isomerase-like protein (DsbD) in Escherichia coli. EMBO J. 14, 3415-3424.

37 Kemmink, J., Darby, N. J., Dijkstra, K., Nilges, M. and Creighton, T. E. (1996) Structure determination of the N-terminal thioredoxin-like domain of protein disulfide isomerase using multidimensional heteronuclear 13C/15N NMR spectroscopy. Biochemistry 35, 7684-7691.

38 Kemmink, J., Darby, N. J., Dijkstra, K., Nilges, M. and Creighton, T. E. (1997) The folding catalyst protein disulfide isomerase is constructed of active and inactive thioredoxin modules. Curr. Biol. 7, 239-245.

39 Stirnimann, C. U., Rozhkova, A., Grauschopf, U., Böckmann, R., Glockshuber, R., Capitani, G. and Grutter, M. G. (2006) High resolution structures of Escherichia coli cDsbD in different redox states: a combined crystallographic, biochemical and computational study. J. Mol. Biol. 358, 829-845.

40 Stirnimann, C. U., Rozhkova, A., Grauschopf, U., Grutter, M. G., Glockshuber, R. and Capitani, G. (2005) Structural basis and kinetics of DsbD-dependent cytochrome $c$ maturation. Structure 13, 953-961.

41 Edeling, M. A., Guddat, L. W., Fabianek, R. A., Thony-Meyer, L. and Martin, J. L. (2002) Structure of CcmG/DsbE at 1.14 A resolution: high-fidelity reducing activity in an indiscriminately oxidizing environment. Structure 10, 973-979. 
42 Goulding, C. W., Apostol, M. I., Gleiter, S., Parseghian, A., Bardwell, J., Gennaro, M. and Eisenberg, D. (2004) Gram-positive DsbE proteins function differently from Gram-negative DsbE homologs. A structure to function analysis of DsbE from Mycobacterium tuberculosis. J. Biol. Chem. 279, 3516-3524.

43 Nakamoto, H. and Bardwell, J. C. (2004) Catalysis of disulfide bond formation and isomerization in the Escherichia coli periplasm. Biochim. Biophys. Acta 1694, 111-119.
44 Lennon, B. W., Williams, C. H. Jr and Ludwig, M. L. (1999) Crystal structure of reduced thioredoxin reductase from Escherichia coli: structural flexibility in the isoalloxazine ring of the flavin adenine dinucleotide cofactor. Protein Sci. 8, 2366-2379.

45 Katti, S. K., LeMaster, D. M. and Eklund, H. (1990) Crystal structure of thioredoxin from Escherichia coli at $1.68 \AA$ resolution. J. Mol. Biol. 212, 167-184.

\section{(1) To access this journal online: (28) http://www.birkhauser.ch}

